Journal of Mathematics and Informatics

Vol. 10, 2017, 113-117

ISSN: 2349-0632 (P), 2349-0640 (online)

Published 11 December 2017

www.researchmathsci.org

DOI: http://dx.doi.org/10.22457/jmi.v10a15

Journal of

Mathematics and

Informatics

\title{
On the Non-Homogeneous Cubic Equation with Four Unknowns $x^{2}-y^{2}=z^{3}+w$
}

\section{S.Dharuna ${ }^{1}$ and D.Maheswari ${ }^{2}$}

Department of Mathematics, Shrimati Indira Gandhi College

Trichy-2, Tamilnadu, India.

1e-mail: $\underline{\text { s_dharuna@yahoo.com; }}$ 'e-mail:matmahes@gmail.com

Received 2 November 2017; accepted 6 December 2017

Abstract. An attempt has been made to determine four non-zero distinct integers $x, y, z$ and $w$ such that the difference of squares of any two integers equals the sum of the cubes of other two integers. A few relations among $x, y, z$ and $w$ are presented. A general formula for generating sequence of integer solutions based on the given solution is also presented.

Keywords: non-homogeneous cubic, cubic with four unknowns, integer solutions

AMS Mathematics Subject Classification (2010): 11D25

\section{Introduction}

Integral solutions for the non-homogeneous Diophantine cubic equation is an interesting concept as it can be seen from [1,2,3]. In [4-8], a few special cases of cubic Diophantine equations with three and four unknowns are studied. In this communication, we present the integral solutions of an interesting cubic equation with four unknowns $x^{2}-y^{2}=z^{3}+w^{3}$. A few remarkable relations between the solutions are presented.

\section{Notations}

$t_{3, n}=\frac{n(n+1)}{2}=$ Triangular number of rank $n$

$t_{4, n}=n^{2}=$ Square number of rank $n$

$t_{6, n}=n(2 n-1)=$ Hexagonal number of rank $n$

$P R_{n}=n(n+1)=$ Pronic number of rank $n$

$G_{n}=2 n-1=$ Gnomonic number of rank $n$

$C t_{m, n}=\frac{m n(n-1)+2}{2}=$ Centered polygonal number of rank $n$ with $m$ sides.

$C P_{n, 6}=n^{3}=$ Centered hexagonal pyramidal number of rank $\mathrm{n}$.

$C P_{n, 5}=\frac{n^{3}+n}{2}=$ Centered pentagonal pyramidal number of rank $\mathrm{n}$ 


\section{S.Dharuna and D.Maheswari}

\section{Method of analysis}

The non-homogeneous cubic equation with four unknowns to be solved is,

$$
x^{2}-y^{2}=z^{3}+w^{3}
$$

Applying the method of factorization, (1) is written as the system of double equations represented by

$$
\begin{aligned}
& x+y=z^{2}-z w+w^{2} \\
& x-y=z+w
\end{aligned}
$$

Solving (2) and (3) for $x$ and $y$, we have

$$
\begin{aligned}
& x=\frac{1}{2}\left(z^{2}-z w+w^{2}+z+w\right) \\
& y=\frac{1}{2}\left(z^{2}-z w+w^{2}-z-w\right)
\end{aligned}
$$

As our interest is on finding integer solutions, we have to choose $z$ and $w$ suitably so that, $x$ and $y$ are integers.

\subsection{Choice}

$$
\text { Let } \left.\begin{array}{rl}
z(k) & =2 k \\
w(l) & =2 l
\end{array}\right\}
$$

Using (6) in (4) and (5)

we have,

$$
\begin{aligned}
& x(k, l)=2 k^{2}-2 k l+2 l^{2}+k+l \\
& y(k, l)=2 k^{2}-2 k l+2 l^{2}-k-l
\end{aligned}
$$

Thus, (6),(7) and (8) are represent integer solutions to (1).

\section{PEOPERTIES}

1. $x(k, l)-y(k, l)$ is always even.

2. $6[x(k, k)+y(k, k)]$ is a Nasty number.

3. $2 x(k, 1)+z(k)-8 t_{3, k}-6 \equiv 0(\bmod 4)$

4. $x(k,-1)-2 P R_{k}+t_{6, k}-2 t_{4, k}-1=0$

5. $y(1, l)+w(l)-t_{6, l}-G_{l} \equiv 0(\bmod 2)$

6. $x(k-1,1)+3 z(k-1)-t_{6, k} \equiv(\bmod 2)$

7. $x(k, k)+w(k)-4 t_{3, k}-G_{k}-1=0$

\subsection{Choice}

Introducing the linear transformations

$$
\left.\begin{array}{l}
x=2 u+v \\
y=2 u-v
\end{array}\right\}
$$

and taking $\left.\quad \begin{array}{rl}z(\mathrm{P}) & =2 \mathrm{P} \\ w(Q) & =2 Q\end{array}\right\}$ 
On the Non-Homogeneous Cubic Equation with Four Unknowns $x^{2}-y^{2}=z^{3}+w$ in (1), it is written as

$$
u v=P^{3}+Q^{3}
$$

which is satisfied by

$$
u=P+Q, v=P^{2}-P Q+Q^{2}
$$

Substituting (11) in (9), we have

$$
\begin{aligned}
& x(P, Q)=2(P+Q)+P^{2}-P Q+Q^{2} \\
& y(P, Q)=2(P+Q)-\left(P^{2}-P Q+Q^{2}\right)
\end{aligned}
$$

Thus, (10),(12) and (13) represent integer solution to (1).

\section{PROPERTIES}
1. $x(P, Q)+y(P, Q)$ is always even.
2. $x(P, P)+4 t_{6, P}-9 t_{4, n}=0$
3. $x(P, 1)-w(P)-P R_{P}-2 C t_{3, P}+3 t_{4, P}-P-1=0$
4. $x(2, P)-y(2, P)-2 P R_{P}+2 G_{P}-6 \equiv 0(\bmod 2)$
5. $y(-1, P)-z(P)+P R_{P}+3=0$
6. $z(P-1)+w(P)-4 P R_{P}+4 t_{4, P}+2=0$
7. $x(P, P)+z(P)-P R_{P}-10 C P_{P, 5}+5 C P_{P, 6}=0$

\subsection{Choice}

Let

$$
\left.\begin{array}{c}
z(k)=2 k+1 \\
w(l)=2 l
\end{array}\right\}
$$

Using (14) in (4) and (5), we have

$$
\begin{aligned}
& x(k, l)=2 k^{2}+2 l^{2}-2 k l+3 k+1 \\
& y(k, l)=2 k^{2}+2 l^{2}-2 k l+k-2 l
\end{aligned}
$$

Thus, (14),(15) and (16) represent integer solutions to (1).

\section{PROPERTIES}

1. $x(k, l)-y(k, l)$ is always odd.

2. $[x(k, l)-y(k, l)-w(l)]^{2}=8 t_{3, k}+1$

3. $x(k, k)+y(k, k)-4 t_{3, k}-t_{6, k}-k-1=0$

4. $x(k, 1)-z(k)-2 P R_{k}-2 \equiv 0(\bmod 3)$

5. $y(k-1,1)+3 w(k-1)-t_{6, k}+3 \equiv 0(\bmod 2)$

6. $6[x(2, k)+y(2, k)-10 k]$ is a Nasty number.

7. $y(k, k)+z(k)-4 t_{3, k}-G_{k}+k=0$

\subsection{Choice}

Let 


$$
\left.\begin{array}{c}
z(k)=(4 k+4) k-5 \\
w(l)=4 l-3
\end{array}\right\}
$$

Using (17) in (1), we have

$$
\begin{aligned}
& x(k, l)=16 k^{6}+48 k^{5}-12 k^{4}-104 k^{3}+16 l^{3}+15 k^{2}-36 l^{2}+75 k+27 l-37 \\
& y(k, l)=16 k^{6}+48 k^{5}-12 k^{4}-104 k^{3}+16 l^{3}+15 k^{2}-36 l^{2}+75 k+27 l-39
\end{aligned}
$$

Thus, (17), (18) and (19) represent integer solutions to (1).

\section{PROPERTIES}

1. $x(k, l)-y(k, l)$ is always two.

2. $z(k)+w(k)-4 P R_{k}-2 G_{k}+6=0$

3. $y(2, k)+z(k)-16 C P_{k, 6}+16 t_{6, n}-1702 \equiv 0(\bmod 15)$

4. $z(k-1)-4 P R_{k}+4 G_{k}+9=0$

5. $w(k)-4 P R_{k}+4 t_{4, k}+3=0$

6. $y(1,-k)+32 C P_{k, 5}+36 P R_{n}+1 \equiv 0(\bmod 25)$

7. $z(k)-w(2 k)-4 P R_{k}+16 C P_{k, 5}-8 C P_{k, 6}+2=0$

\section{Generation of solutions}

Let $\left(x_{0}, y_{0}, z_{0}, w_{0}\right)$ be the given initial integer solution of (1).

Let $x_{1}=2 h-3^{3} x_{0}, y_{1}=h+3^{3} y_{0}, z_{1}=3^{2} z_{0}, w_{1}=3^{2} w_{0}$

be the second solution of (1), where $\mathrm{h}$ is a non-zero integer to be determined.

Substituting (20) in (1) and simplifying, we get

$$
h=36 x_{0}+18 y_{0}
$$

Therefore, the second solution of (1) expressed in the matrix form is,

$$
\left(x_{1}, y_{1}\right)^{t}=\mathrm{M}\left(x_{0}, y_{0}\right)^{t}, \quad z_{1}=3^{2} z_{0}, w_{1}=3^{2} w_{0}
$$

where, $\mathbf{M}=\left[\begin{array}{ll}45 & 36 \\ 36 & 45\end{array}\right]$

Repeating the above process, we have, in general

$$
\left(x_{n}, y_{n}\right)^{t}=\mathrm{M}^{n}\left(x_{0}, y_{0}\right)^{t}, \quad z_{n}=3^{2 n} z_{0}, w_{n}=3^{2 n} w_{0}
$$

where, $\quad \mathbf{M}^{n}=\frac{9^{n}}{2}\left[\begin{array}{ll}9^{n}+1 & 9^{n}-1 \\ 9^{n}-1 & 9^{n}+1\end{array}\right]$

Giving $n=1,2,3, \ldots$ inturn in (21), one obtains sequence of integer solutions to

(1) based on the given solution $\left(x_{0}, y_{0}, z_{0}, w_{0}\right)$.

\section{Conclusion}

In this paper, we have presented infinitely many non-zero distinct solutions to the nonhomogeneous cubic equation with four unknowns given by $x^{2}-y^{2}=z^{3}+w^{3}$. In other words, this problem under consideration is equivalent to finding non-zero distinct integer 
On the Non-Homogeneous Cubic Equation with Four Unknowns $x^{2}-y^{2}=z^{3}+w$

quadruples such that the difference of squares of any two members in a quadruple equals the sum of the cubes of other two member of the quadruple. In conclusion, one may search for quadruples with different relations among its members.

\section{REFERENCES}

1. L.E.Dickson, History of Theory of Numbers, Vol 2, Chelsea publishing company, New York, (1952).

2. L.J.Mordell, Diophantine Equations, Academic press, London, (1969).

3. R.D.Carmichael, The theory of numbers and Diophantine analysis, New York, Dover,(1959).

4. M.A.Gopalan and S.Premalatha, Integral solutions of $(x+y)\left(x y+w^{2}\right)=2\left(k^{2}+1\right) z^{3}$. Bulletin of Pure and Applied Sciences, 28E (2009) 197-202.

5. M.A.Gopalan and V.Pandichelvi, Remarkable solutions on the cubic equation with four unknowns $x^{3}+y^{3}+z^{3}=28(x+y+z) w^{2} \quad$ Antarctica J. of Maths., 4(4) (2010) 393-401.

6. M.A.Gopalan and B.Sivagami, Integral solutions of homogeneous cubic equation with four unknowns $x^{3}+y^{3}+z^{3}=3 x y z+2(x+y) w^{3}$, Impact. J. Sci. Tec, 4(3) (2010) 53-60.

7. M.A.Gopalan and S.Premalatha, On the cubic Diophantic equations with four unknowns $(x-y)\left(x y-w^{2}\right)=2\left(n^{2}+2 n\right) z^{3}$, International Journal of Mathematical Sciences, 9(1-2) ( (2010) 171-175.

8. M.A.Gopalan and J.Kaliga Rani, Integral solutions of $x^{3}+y^{3}+(x+y) x y=z^{3}+w^{3}+(z+w) z w$, Bulletin of Pure and Applied Sciences, 29E (1) (2010) 169-173. 